\title{
Evaluation of gastrointestinal injury in blunt abdominal trauma "FAST is not reliable": the role of repeated ultrasonography
}

\author{
Afshin Mohammadi ${ }^{1 *}$ and Mohammad Ghasemi-rad ${ }^{2}$
}

\begin{abstract}
Background: To determine the diagnostic Accuracy of Focused Assessment Sonography for Trauma (FAST) and repeated FAST in the patients with blunt abdominal trauma.

Methods: In this retrospective study we collected the data of all patients from September 2007 to July 2011 with gastrointestinal injury. The intraoperative outcome was compared with FAST technique and the repeated or delayed sonography.

Results: A total number of 1550 patients with blunt abdominal trauma underwent FAST in a period of 4 years in our hospital. Eighty-eight (5.67\%) patients were found to have gastrointestinal injury after exploratory laparotomy. Fifty-five (62.5\%) patients had isolated gastrointestinal injury and 33 (37.5\%) patients had concomitant injury to the other solid organs. In those with isolated gastrointestinal injury, the sensitivity of FAST was 38.5\%. Repeated ultrsonography was performed in 34 patients with false negative initial FAST after 12-24 hours. The sensitivity of repeated ultrasonography in negative initial FAST patients in detection of gastrointestinal injury was $85.2 \%$ (95\% Cl, $68.1 \%$, and $94.4 \%)$.
\end{abstract}

Conclusion: Repeated sonography after 12 to 24 hours in patients with negative initial FAST but sustain abdominal symptom can facilitated a diagnosis of Gl tract injury and can be as effective method instead of Computed tomography in developing country.

Keywords: Ultrasonography, Gastrointestinal, Trauma, FAST

\section{Background}

Trauma is the most common cause of mortality in 1-45 year's age group [1]. Currently ultrasonography (US) is the primary method of screening patients with blunt abdominal trauma (BAT) worldwide [1-3]. Focused Assessment Sonography for Trauma (FAST) has been previously described for the evaluation of blunt abdominal trauma to observe the presence of free fluid in the abdomen or pelvis [4].

Although in some of the previous published literature they believe that it is rare to see false-negative results when screening with US $(1 \%)[5,6]$. It seems that screening BAT with FAST will lead to under diagnosis in

\footnotetext{
*Correspondence: Mohamadi_Afshin@yahoo.com

'Department of Radiology, Urmia University of Medical Sciences, Urmia,

West-Azerbaijan, Iran

Full list of author information is available at the end of the article
}

some abdominal injuries such as; retroperitoneal (pancreatic and adrenal), vascular injuries and diaphragmatic rupture that may have a negative impact on the patients outcome [7].

Due to subtle findings FAST has been reported to be of less value in detection of bowel and mesenteric injuries [8]. Although it is uncommon to develop hollow visceral organ injury after BAT but they are very important to diagnose, because there is no conservative treatment for these types of injuries and all of the patients with such injuries even in unequivocal cases, they need to undergo operative intervention [9]. According to the previous reports the morbidity of gastrointestinal tract injury is mostly related to delays diagnosis [10].

Because of less availability of computed tomography in developing country, the purpose of our study was to determine the role of repeated abdominal US in the

\section{() Biomed Central}


patients with negative "FAST "to early diagnose hollow viscous organ injury in patients with BAT. To our best knowledge this is the first report evaluating the role of repeated abdominal sonography to determine and reduce missed gastrointestinal injury by FAST technique.

\section{Methods}

This retrospective study was started from September 2007 to July 2011. On thousand five hundred and fifty emergency ultrasonography with FAST technique were performed in our University hospital in order to detect free intra-abdominal fluid as an indicator of intra-abdominal organ injury in-patient with BAT (Figure 1, 2).

The outcome of FAST technique and the data regarding type of abdominal injuries were obtained by retrospectively going through patient's operation notes. After retrospectively reviewing the operation record of 1550 BAT patients, 88 were found to have gastrointestinal injury. This study was performed in Imam training University Hospital that serves as the only trauma referral center in our provenance. University review board and ethic committee approved the study.

All the injured patients were referred to our center, maximum one hour after trauma and US examination was performed during first 30 minutes of admission. Examination was performed by one radiologist in the department of radiology at the emergency room. FAST technique was performed by using Sonoline G 40 ultrasound devise (Siemens, Germany) with 3.5-5 MHZ convex transducer. Six areas of the abdomen were examined to detect free fluid; left upper quadrant (LUQ), Morrison pouch, right upper quadrant (RUQ), pelvis, right and left para-colic gutters.

Abdomen and pelvic spiral Computed Tomography (CT) Scan examinations were performed only with IV contrast (Toshiba; X-vision scanner) in 39 patients with BAT and negative FAST after 12-24 hours due to worsening of clinical problem but stable hemodynamic condition.

Spiral CT scans were performed with $10-\mathrm{mm}$ collimation and a table speed of $10 \mathrm{~mm} / \mathrm{sec}$. Images were reconstructed at $7-\mathrm{mm}$ intervals. In adults, a total of $120 \mathrm{ml}$ of Iohexol (Omnipaque, $300 \mathrm{mg} / 50 \mathrm{cc}$ ) was administered intravenously at a rate of $3-4 \mathrm{ml} / \mathrm{sec}$. Another experienced radiologist interpreted all of the abdominal CT scans.

The routine protocol in our center is that every patient with suspected abdominal trauma should undergo FAST. Except for those patients that further delaying to intervene to undergo FAST is not possible and the patients need to directly go to the operation room. Those patients with unstable hemodynamics and observable fluid in the peritoneal cavity should immediately undergo laparotomy. Patients with stable hemodynamics and positive sonography will undergo conservative management and close observation.

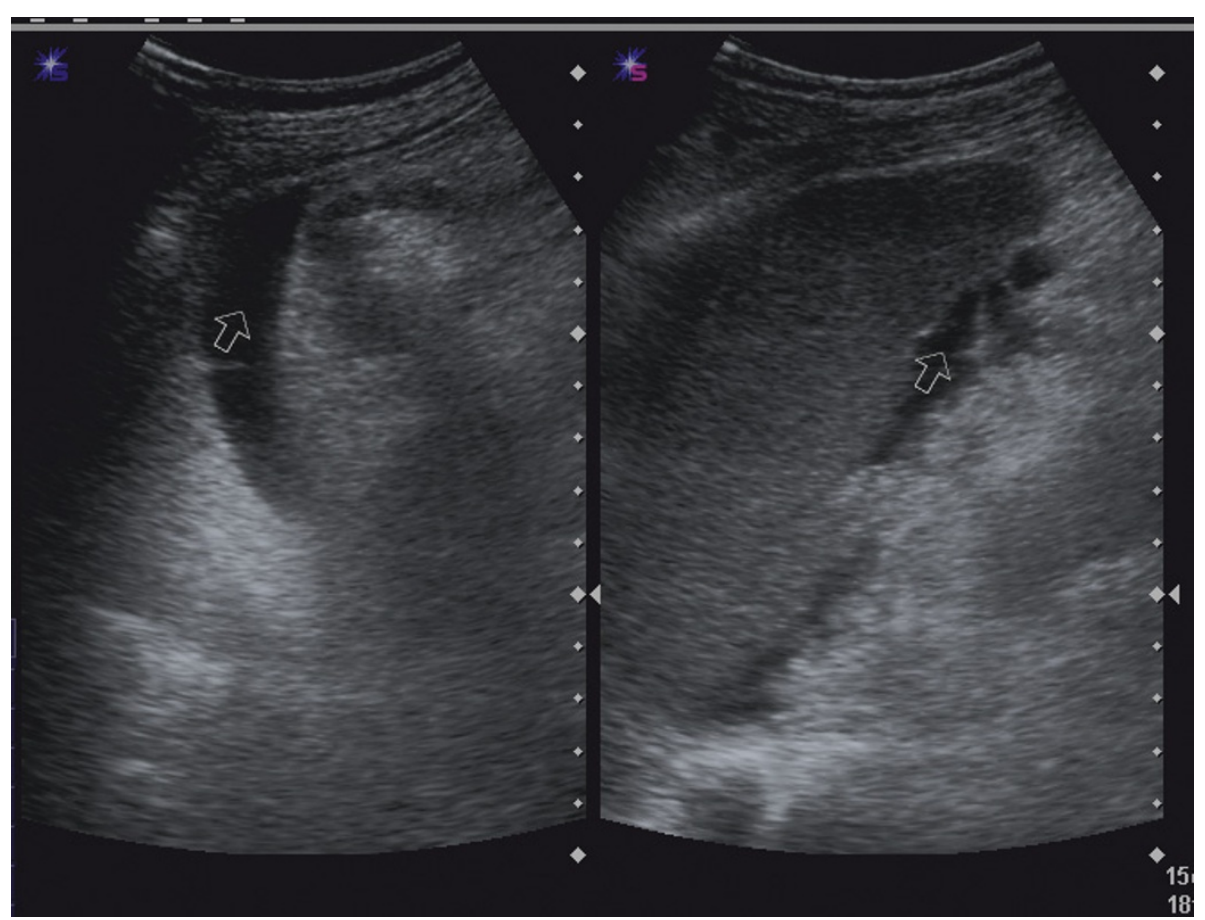

Figure 1 Longitudinal sonogram show free fluid (arrow) associated with lleal perforation in pelvic cavity. 


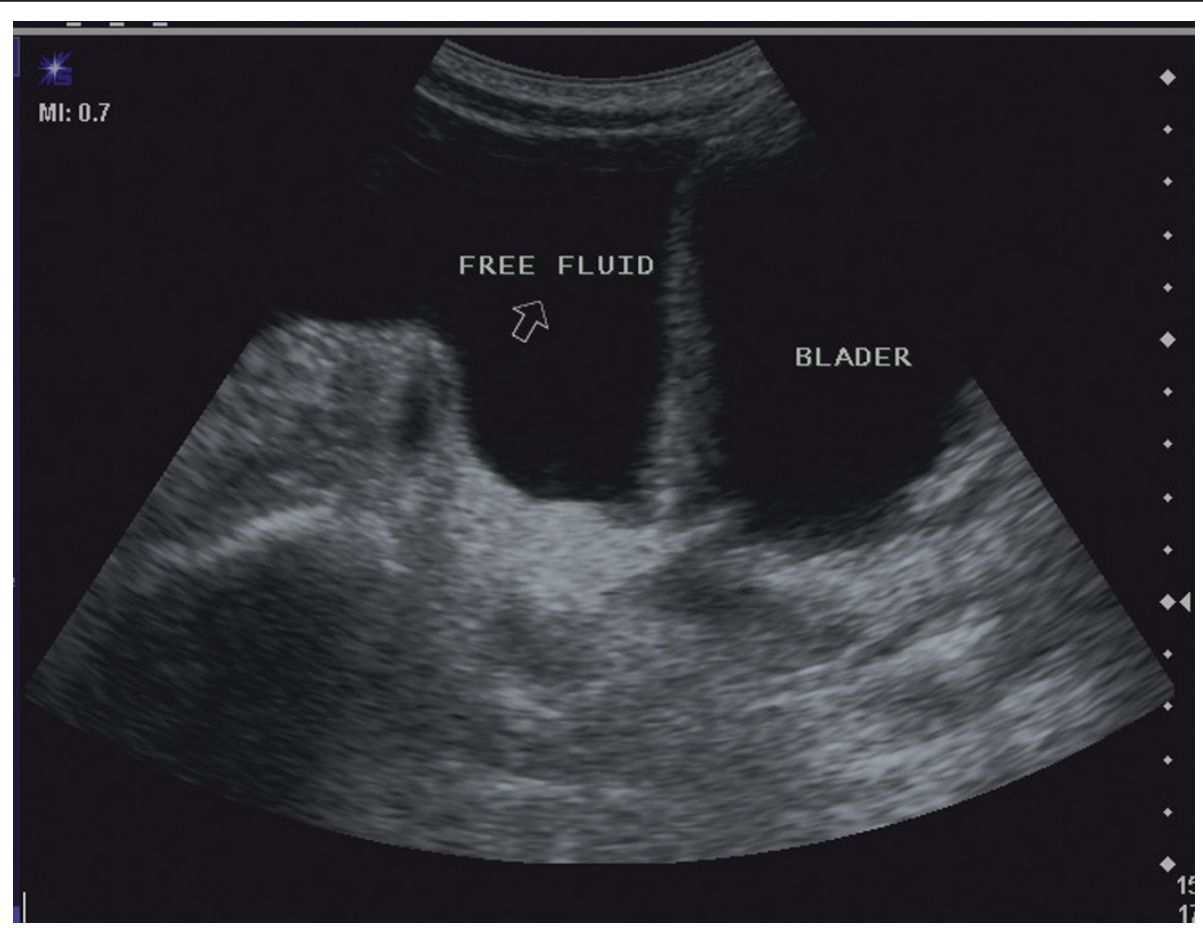

Figure 2 Ultrasonogram revealed free fluid in the paracolic gutter (right) and perisplenic (left).

Those with negative clinical signs and negative FAST are not followed by any other diagnostic methods. But in those patients with negative FAST and constant abdominal pain and stable hemodynamic due to shortage of intravenous contrast material in our center they have to undergo repeated FAST after 12 to 24 hours.

The results of FAST technique were compared with surgical results. Statistical analysis was performed to determine the sensitivity and $95 \%$ confidence interval were calculated and used for determining the diagnostic accuracy.

\section{Results}

Out of 1550 patients with BAT a total number of 352 patients (44\%) underwent operation. Eighty- eight $(5.67 \%)$ patients had gastrointestinal injury in exploratory laparotomy (66 (75\%) were male and $22(25 \%)$ were female).

The mean age was $28.9 \pm 16.5$ years (Age range: $3-80$ Years). Seventy-one (80.6\%) patients had abdominal tenderness during primary physical examination. Fortyseven (53\%) patients had stable hemodynamic condition and $41(46.5 \%)$ patients were hypotensive at the time of US examination.

Fifty-five (62.5\%) patients had isolated gastrointestinal injury and $33(37.5 \%)$ patients had concomitant injury to the other solid organ such as spleen $(n=14)$, liver $(\mathrm{n}=13)$, Diaphragm $(\mathrm{n}=2)$, Pancreas $(\mathrm{n}=2)$ and kidney $(\mathrm{n}=2)$.

Emergency US with FAST technique was positive for free fluid in $49(55.6 \%)$ patients (True positive) and was negative (false negative) in 39 (44.3\%) patients with gastrointestinal injury.

From 49 patients with true positive FAST, 28 (57.1\%) patients had solid organ injury concomitant with bowel injury and $21(42.8 \%)$ patients had isolated gastrointestinal injury. A total of 55 (62.5\%) out of 88 patients had isolated bowel injury; FAST exam was positive only in $21(38.1 \%)$ patients (True positive) and was negative in $34(61.8 \%)$ patients. In 34 patients with isolated gastrointestinal injury FAST was negative for free fluid (False negative).

In $39(44.3 \%)$ patients with BAT that the result of emergency US did not show free intra peritoneal fluid in 34 patients, the underwent conservative management and after 12-24 hours serial physical examination showed abdominal tenderness and guarding and worsening of abdominal pain. Upon repeated ultrasonography there was free intra-peritoneal fluid in 29 patients and negative results in 10 patients. All those patients (39 patients) underwent abdominal and pelvic CT, which revealed hollow viscous organ injury in 24 (61.5\%) patients. In 15 (38.4\%) patients CT examination did not show gastrointestinal injury (false negative) all 
of which underwent surgical operation because of sustained guarding and unstable hemodynamic condition.

The sensitivity of FAST for detection of gastrointestinal injury in those patients with isolated gastrointestinal injury, the sensitivity was $38.5 \%$ (95\% CI, $23.2 \%$, and $53.7 \%)$.

From 34 patients with negative initial FAST the repeated ultrasonography revealed free fluid in 29 patients and was negative in 5 patients then the sensitivity of repeated ultrasonography in negative initial FAST in detection of gastrointestinal injury was $85.2 \%$ (95\% CI, 68.1\%, and 94.4\%).

The sensitivity of CT for the detection of specific sign of gastrointestinal injury such as free air and bowel thickening in the entire study group was $61.5 \%$ (95\% CI, $.44 .6 \%, 76.1 \%)$.

The distribution of gastrointestinal injury in these 88 patients is presented in table 1 and distribution of concomitant solid organ injury is presented in table 2 .

\section{Discussion}

Rapid diagnosis and treatment of abdominal injury is an important step to prevent death in BAT patients [1].

Physical examination is frequently unreliable in the setting of acute trauma [11].

Many of the previous reports show that emergency ultrasound is effective in diagnosis of hemo-peritoneum [1,12-14]. Now FAST technique has gained popularity and is been accepted as a diagnostic modality for evaluation of patients with trauma $[1,10-15]$. Our previous experience showed that sensitivity of FAST in the diagnosis of BAT is $95.4 \%[1]$.

MacGahan et al reported free fluid in only three patients with isolated bowel and mesenteric injury in a series of 500 trauma patients [7]. There are several articles pointing that some important abdominal organ injury can be missed by ultrasonography. Dolich et al reported a large number of abdominal injuries (33\%), which required operation and were missed in US examination [16].

Table 1 table shows the distribution of gastrointestinal injury in trauma

\begin{tabular}{ccc}
\hline Location & Number & Total \\
\hline Small bowel & 7 & 71 \\
Duodenum & 36 & \\
Jejunum & 28 & \\
Ileum & & 17 \\
Large bowel & 3 & \\
Ascending colon & 10 & \\
Sigmoid colon & 4 & \\
Transverse colon & & \\
\hline
\end{tabular}

Table 2 table shows the distribution of concomitant solid organ injury is trauma patients

\begin{tabular}{ll}
\hline Location & Number \\
\hline Spleen & 14 \\
Liver & 13 \\
Kidney & 2 \\
Diaphragm & 2 \\
Pancreas & 2 \\
\hline
\end{tabular}

Shanmuganathan et al showed that 34\%(157 patients) of 467 patients with BAT had no free fluid in emergency US [13]. He studied more than 11,000 patients with BAT and concluded that the FAST technique may frequently miss patients with surgically correctable injuries.

Previous reports are indicative of a limited value for FAST in the diagnosis of certain type of injuries such as; diaphragmatic rupture [17], pancreatic [15] and mesenteric injury [18-20].

MacGahan JP et al demonstrated a sensitivity of $44 \%$ for diagnosis of isolated gastrointestinal injury by FAST [21]. They also showed that free abdominal fluid was not detected in the majority of patients with isolated bowel and mesenteric injury. Observation, serial physical abdominal examination, Clinical suspicion for bowel and mesenteric injury and CT can all be of help to diagnose intra-abdominal organ injuries.

In our study 39 patients with negative initial US examination and persistent abdominal pain and tenderness underwent repeated ultrasonography after a period of 12-24 hours. Repeated US detected free intra-peritoneal fluid in 29 patients.

Diagnosing gastrointestinal trauma is difficult based on emergency rooms physical examination [19-21] and necessitates using other imaging modality such as CT scan [22,23].

$\mathrm{CT}$ has been reported to have a sensitivity ranging from $93-100 \%$ in detection of bowel and mesenteric injury. Mirvis et al prospectively detected bowel and mesenteric injury in $17(100 \%)$ patients undergoing laparotomy [22].

Atri et al showed that sensitivity of the three observers in diagnoses of surgically important bowel or mesenteric injury by CT scan ranged from $87 \%-95 \%$ [23]. They concluded that multi-detector CT has high negative predictive value and can accurately show important bowel or mesenteric injuries.

Levine et al [24] reported that only bowel wall thickening and free air were specific finding in the CT scanning (Figure 3).

And other sign such as, free fluid are nonspecific not reliable to differentiate between bowel and solid organ injuries. 


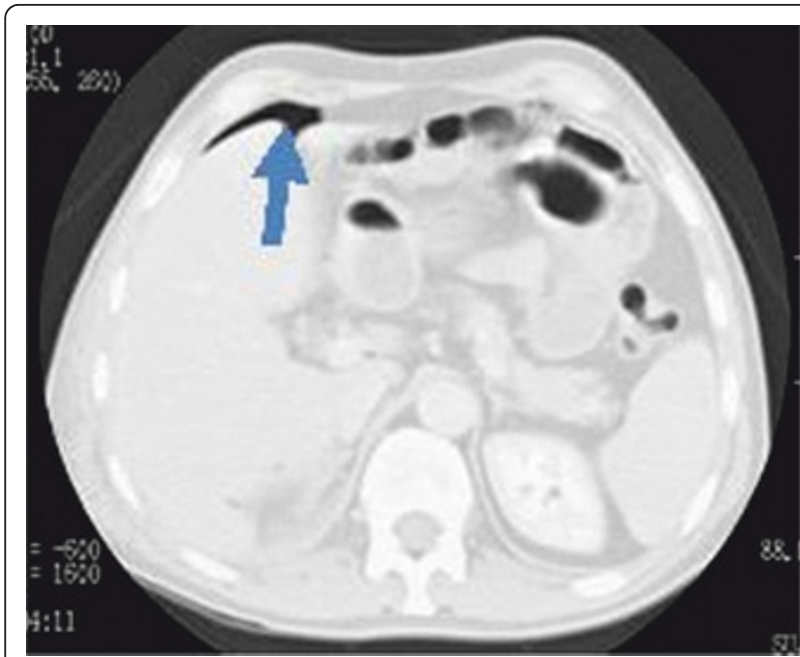

Figure 3 Abdominal CT scan with lung window shows free air adjacent to liver edge due to colon perforation.

The sensitivity of CT for diagnosis of gastrointestinal trauma in our study is lower compare to other studies $[22,23,25]$, because they used multi-detector CT that is more accurate in diagnosis of GI tract pathology.

McGahan JP et al reported that $49 \%$ of the patients with gastrointestinal injury had concomitant injury to other solid organs. The results of our study showed that $38 \%$ patients with blunt abdominal trauma had concomitant solid organ injury.

In our study jejunum and ileum were the most common sites of gastrointestinal trauma respectively. The most common solid organ injury concomitant with gastrointestinal trauma was spleen followed by the liver, which were similar to the report by Richards JL et al [18].

The limitations of our study are; single detector CT which can miss some of the intra-abdominal injuries, the retrospective part of the study which we might have missed some of the data in the records, patients with subtle injury such as mild intestinal hematoma may not show clinical symptom and could be missed because they did not underwent repeated abdominal sonography, Inability to calculate the specificity, positive predictive and negative predictive value Since the small injuries could not be seen and consequently are not going to the be operated on.

It is difficult to diagnose gastrointestinal trauma when FAST is performed immediately after admission. As is shown in our report only $38.5 \%$ of the patients with free fluid in the abdomen on initial FAST had isolated gastrointestinal trauma. We recommend performing a serial US when CT is not available in-patient suspected of GI trauma and persistent abdominal pain and tenderness, which can reduce the risk of missing major intraabdominal injuries.

List of abbreviations

CT: Computed tomography; FAST: Focused Assessment of Sonography for Trauma.

\section{Acknowledgements}

Urmia University of Medical Sciences supported this research.

\section{Author details}

'Department of Radiology, Urmia University of Medical Sciences, Urmia, West-Azerbaijan, Iran. ${ }^{2}$ Student research committee, Urmia University of medical Sciences. Urmia, Iran.

\section{Authors' contributions}

All the authors in this manuscript have read and approve the final manuscript. AM: Concept, design and the Ultrasonographic studies MG: Manuscript writing and editing and Data analysis.

\section{Competing interests}

The authors declare that they have no competing interests.

Received: 15 December 2011 Accepted: 20 January 2012

Published: 20 January 2012

\section{References}

1. Mohammadi A, Daghighi MH, Poorisa M, Afrasiabi K, Pedram A: Diagnostic Accuracy of Ultrasonography in Blunt Abdominal Trauma. Iran J Radiol 2008, 5(3):135-139.

2. Brown MA, Casola G, Sirlin CB, Budorick N, Patel N, Hoyt DB: Blunt abdominal trauma: screening US in 2,693 patients. Radiology 2001, 218:352-358.

3. Brown MA, Sirlin CB, Hoyt DB, Casola G: Screening ultrasound in blunt abdominal trauma. J Intensive Care Med 2003, 18:253-260.

4. McGahan JP, Richards J, Gillen M: The focused abdominal sonography for trauma scan: pearls and pitfalls. J Ultrasound Med 2002, 21:789-800.

5. Pinto F, Bignardi E, Pinto A, Rizzo A, Scaglione M, Romano L: Ultrasound in the triage of patients after blunt abdominal trauma: our experience in 3,500 consecutive patients. Radiology 2002, 225:358.

6. Sirlin CB, Casola G, Brown MA, Patel N, Bendavid EJ, Hoyt DB: Quantification of fluid on screening ultrasonography for blunt abdominal trauma: a simple scoring system to predict severity of injury. J Ultrasound Med 2001, 20:359-366.

7. McGahan JP, Rose J, Coates TL, Wisner DH, Newberry P: Use of sonography in the patient with acute abdominal trauma. J Ultrasound Med 1997, 16:653-662.

8. Lee BC, Ormsby EL, McGahan JP, Melendres GM, Richards JR: The utility of sonography for the triage of blunt abdominal trauma patients to exploratory laparotomy. AJR Am J Roentgenol 2007, 188(2):415-21.

9. Hughes TM: The diagnosis of gastrointestinal tract injuries resulting from blunt abdominal trauma. Aust NZ J Surg 1999, 69:770-777.

10. Wisner DH, Chun Y, Blaisdell FW: Blunt intestinal injury. Arch Surg 1990, 125:1319-23.

11. Schurink GW, Bode PJ, van Luijt PA, van Vugt AB: The value of physical examination in the diagnosis of patients with blunt abdominal trauma: a retrospective study. Injury 1997, 28:261-265.

12. McKenney M, Lentz $K$, Nunez $D$, et al: Can Ultrasound replace diagnostic peritoneal lavage in the assessment of blunt trauma? J Trauma 1994, 37:439-441.

13. Shanmuganathan $\mathrm{K}$, Mirvis SE, Sherbourne CD, Chiu WC, Rodriguez A: Hemoperitoneum as the sole indicator of abdominal visceral injuries: a potential limitation of screening abdominal US for trauma. Radiology 1999, 212:423-430

14. Bode PJ, Edwards MJR, Kruit MC, Van Vugt AB: Sonography in a clinical algorithm for early evaluation of 1671 patients with blunt abdominal trauma. AJR Am J Roentgenol 1999, 172:905-911. 
15. McGahan JP, Richards JR: Blunt abdominal trauma: the role of emergent sonography and a review of the literature. AJR Am J Roentgenol 1999, 172:897-930.

16. Dolich MO, McKenney MG, Varela JE, Compton RP, McKenney KL, Cohn SM: 2,576 ultrasounds for blunt abdominal trauma. J Trauma 2001, 50:108-112.

17. Simpson J, Lobo DN, Shah AB, Rowlands BJ: Traumatic diaphragmatic rupture: associated injuries and outcome. Ann R Coll Surg Engl 2000, 82:97-100.

18. Richards JR, McGahan JP, Simpson JL, Tabar P: Bowel and mesenteric injury: evaluation with emergency abdominal US. Radiology 1999, 211:399-403.

19. Bensard DD, Beaver BL, Besner GE, Cooney DR: Small bowel injury in children after blunt abdominal trauma: is diagnostic delay important? J Trauma 1996, 41:476-483.

20. Burney RE, Mueller $\mathrm{GL}$, Coon $\mathrm{GL}$, et al: Diagnosis of isolated small bowel injury following blunt abdominal trauma. Ann Emerg Med 1983, 12:71-74.

21. Bloom Al, Rivkind A, Zamir $G$, et al: Blunt injury of the small intestine and mesentery: the trauma surgeon's Achilles heel? Eur J Emerg Med 1996, 3:85-91.

22. Mirvis $S E$, Gens $D R$, Shanmuganathan $K$ : Rupture of the bowel after blunt abdominal trauma: diagnosis with CT. AJR 1992, 159:1217-1223.

23. Atri M, Hanson JM, Grinblat L, Brofman N, Chugtai T, Tomlinson G: Surgically important bowel and/or mesenteric injury in blunt trauma: accuracy of multidetector CT for evaluation. Radiology 2008, 249(2):524-33.

24. Levine CD, Gonzales RN, Wachsberg RH, Ghanekar D: CT findings of bowel and mesenteric injury. J Comput Assist Tomogr 1997, 21(6):974-9.

25. Breen DJ, Janzen DL, Zwirewich CV, Nagy AG: Blunt bowel and mesenteric injury:diagnostic performance of CT sings. J Comput Assist Tomogr 1997, 21:706-712.

doi:10.1186/1749-7922-7-2

Cite this article as: Mohammadi and Ghasemi-rad: Evaluation of gastrointestinal injury in blunt abdominal trauma "FAST is not reliable": the role of repeated ultrasonography. World Journal of Emergency Surgery 2012 7:2.

\section{Submit your next manuscript to BioMed Central} and take full advantage of:

- Convenient online submission

- Thorough peer review

- No space constraints or color figure charges

- Immediate publication on acceptance

- Inclusion in PubMed, CAS, Scopus and Google Scholar

- Research which is freely available for redistribution 